Mesilato de Imatinibe: Uma Otimização em sua Síntese

\author{
Boechat, N.;* Bastos, M. M.; Duarte, S. L.; Costa, J. C. S.; Mafra, J. C. M.; \\ Daniel, L. C. C.
}

Rev. Virtual Quim., 2013, 5 (2), 222-234. Data de publicação na Web: 5 de janeiro de 2013

http://www.uff.br/rvq

\title{
Imatinib Mesylate: An Optimization in its Synthesis
}

\begin{abstract}
Imatinib mesylate - gleevec ${ }^{\circledR}$ is considered a major alternative for the treatment of chronic myelogenous leukemia. Clinical studies demonstrate a complete hematological response in most patients treated with this drug. The effectiveness of the drug, the high cost and the patent expiration has been responsible for the publication of numerous studies that seek to improve the synthesis of this drug. This study reports an optimization of the first synthetic process described in the literature. Batches were synthesized on a semi-pilot scale, to develop methodologies that may be available for the production of the product on an industrial scale.
\end{abstract}

Keywords: Gleevec ${ }^{\circledR}$; imatinib mesylate; cancer; kinase tirosine; leukemia.

\section{Resumo}

O mesilato de imatinibe - glivec $^{\circledR}$ é considerado como uma das principais alternativas para o tratamento da leucemia mieloide crônica. Estudos clínicos demonstram uma resposta hematológica completa em grande parte dos pacientes tratados com este medicamento. A eficácia do fármaco, o seu alto custo e a queda de sua patente são fatores responsáveis pelo desenvolvimento de processos que buscam melhorar a sua síntese. Este trabalho descreve a otimização do primeiro processo sintético descrito na literatura. Foram sintetizados lotes em escala semi-piloto, visando desenvolver metodologias que possam estar disponíveis para a produção do medicamento em escala industrial.

Palavras-chave: Glivec $^{\circledR}$; mesilato de imatinibe; cancer; tirosina quinase; leucemia.

\footnotetext{
* Fundação Oswaldo Cruz, Instituto de Tecnologia em Fármacos, Departamento de Síntese Orgânica, Manguinhos, CEP 21041-250, Rio de Janeiro-RJ, Brasil.

M boechat@far.fiocruz.br

DOI: $\underline{10.5935 / 1984-6835.20130023}$
} 


\title{
Mesilato de Imatinibe: Uma Otimização em sua Síntese
}

Núbia Boechat, ${ }^{\mathrm{a}, *}$ Mônica M. Bastos, ${ }^{\text {a }}$ Silvio Luiz Duarte, ${ }^{\mathrm{a}}$ Jorge Carlos dos S. Costa, ${ }^{a}$ João Carlos M. Mafra, ${ }^{a}$ Lucilene da C. C. Daniel ${ }^{a, b}$

\author{
${ }^{a}$ Fundação Oswaldo Cruz, Instituto de Tecnologia em Fármacos, Departamento de Síntese \\ Orgânica, Manguinhos, CEP 21041-250, Rio de Janeiro-RJ, Brasil. \\ ${ }^{\text {b}}$ Centro Universitário UNIABEU, Rua Itaiara, 301, CEP 26113-400, Belford Roxo-RJ, Brasil. \\ * boechat@far.fiocruz.br
}

Recebido em 26 de outubro de 2012. Aceito para publicação em 13 de dezembro de 2012

\section{Introdução \\ 2. Resultados e Discussão \\ 3. Conclusão \\ 4. Experimental}

\section{Introdução}

Segundo os dados de 2012, o câncer é uma das causas principais de óbitos da história da humanidade, sendo o responsável por $13 \%$ das mortes por ano no mundo. ${ }^{1}$ Nas últimas décadas, o câncer tornou-se um problema de saúde pública mundial. A Organização Mundial da Saúde (OMS) estima que, no ano 2030, 27 milhões de casos incidentes de câncer, 17 milhões de mortes e 75 milhões de pessoas vivendo com a doença, sendo a maior ocorrência em países de renda baixa e média. ${ }^{2}$ No Brasil, as previsões para o ano de 2012, válidas também para o ano de 2013, são de 257.870 casos novos para o sexo masculino e 260.640 para o sexo feminino. Estima-se que os tipos de câncer mais incidentes na população brasileira serão o de próstata $(60 \mathrm{mil})$, mama feminina (53 mil), cólon e reto $(30 \mathrm{mil})$, pulmão (27 mil), estômago (20 mil) e colo do útero (18 mil). ${ }^{2,3}$

A leucemia mieloide crônica (LMC) é uma neoplasia que se caracteriza como uma desordem clonal de células-tronco hematopoiéticas. Esta desordem do sistema hematológico está diretamente associada à presença de um cromossomo específico, designado Filadélfia $(\mathrm{Ph})$, que é detectado em 95\% dos pacientes com LMC. $^{4}$ Este cromossomo é formado a partir da quebra dos cromossomos 22 e $9 .^{5}$ A LMC corresponde a $15 \%$ de todos os novos casos de leucemia no Brasil e ocorre mais comumente em indivíduos com idade avançada, com média entre 45 a 55 anos ou com idade igual ou superior a 60 anos. ${ }^{2-4}$

O cromossomo Ph leva a formação de uma proteína, denominada de tirosina quinase, que regula uma série de processos celulares, incluindo crescimento e sobrevivência. A atividade desregulada destas enzimas é um dos fatores responsáveis pelas transformações malignas 
em neoplasmas. ${ }^{6,7}$

Yaish e colaboradores ${ }^{8}$ descreveram o primeiro inibidor sintético da tirosina quinase, a tirfostina (1). Desde então, foram realizadas várias pesquisas que mostraram o elevado potencial do esqueleto 2fenilaminopirimidina (FAP) (2) como um inibidor não específico de quinase (Figura 1). ${ }^{9-13}$<smiles>N#CC(C#N)=Cc1cc(O)c(O)c(O)c1</smiles>

Tirfostina (1)

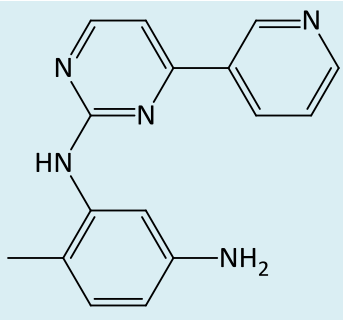

Esqueleto FAP (2)

Figura 1. Estrutura da tirfostina e do esqueleto FAP

O mesilato de imatinibe (Figura 2) (glivec $^{\circledR}$, STI571 - inibidor de transdução de sinal) (3) foi desenvolvido com base em estudos de relação estrutura-atividade (REA/SAR), como um inibidor específico do receptor do fator de crescimento de plaquetas, mas mostrou-se um potente inibidor da tirosina quinase. Ele age nesta proteína e causa a morte das células anormais por ela produzidas. ${ }^{6-7}$

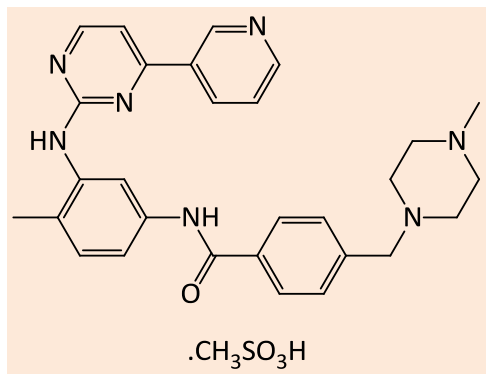

(3)

Figura 2. Estrutura do glivec ${ }^{\circledR}$

O glivec ${ }^{\circledR}$ foi desenvolvido pela Novartis e licenciado, em 7 de novembro de 2001, para tratamento de pacientes com LMC pelo Food and Drug Administration (FDA). Ele representa uma evolução na história do tratamento para pacientes portadores de LMC e, nos últimos anos, as pesquisas sobre a síntese do mesilato de imatinibe aumentaram devido à importância deste fármaco no cenário mundial. Nos Estados Unidos, após alguns testes clínicos, foi anunciada a decisão de utilizar este medicamento para o tratamento de um tipo raro de câncer gastrointestinal, chamado tumor estromal gastrintestinal (GIST). ${ }^{14}$

O mesilato de imatinibe foi sintetizado pela primeira vez, em 1993, por Zimmermann. $^{15}$ A rota sintética original desenvolvida por este pesquisador apresenta uma série de problemas, como a manipulação de reagentes tóxicos e etapas de reação sem a descrição dos rendimentos. ${ }^{16,17}$

Desde a descoberta do imatinibe, inúmeros trabalhos foram descritos na literatura com o objetivo de obter esta substância com a melhor relação custo/benefício. ${ }^{18-28}$ No entanto, os processos ainda apresentam uma série de limitantes. Além disso, a expiração da patente despertou o interesse das farmoquímicas para o desenvolvimento de um processo eficaz para a produção do ingrediente farmacêutico ativo (IFA).

O governo, através do Ministério da Saúde (MS), criou a Política Nacional de Atenção Oncológica (PNAO) que contempla ações de promoção, prevenção, diagnóstico, tratamento, reabilitação e cuidados 
paliativos, a ser implantada em todas as unidades federadas. Desta forma, o desenvolvimento tecnológico de fármacos antineoplásicos torna-se imperativo, não só para auxiliar na negociação de preços com os grandes laboratórios, mas também em caso de desabastecimento, suprindo as demandas do Estado. Devido a este fato, o MS publicou a portaria 1284, de 26 de maio de 2010, criando a relação de medicamentos essenciais - RENAME, que elenca 87 produtos estratégicos (fármacos/medicamentos) no âmbito do Sistema Único de Saúde (SUS), com intuito de colaborar com o Complexo Industrial de Saúde (CIS). Sabe-se que estes produtos contribuem para o aumento do déficit da balança comercial brasileira (cerca de 10 bilhões de dólares). 0 glivec ${ }^{\circledR}$ é um destes 87 produtos estratégicos, e faz parte de uma Parceria de Desenvolvimento Produtivo (PDP) firmado entre Farmanguinhos/FIOCRUZ e Cristália Produtos Químicos Farmacêuticos Ltda. $^{29}$

O presente trabalho teve como objetivo o desenvolvimento do ingrediente farmacêutico ativo (IFA) mesilato de imatinibe em escala de laboratório e piloto, além da otimização do processo original descrito por Zimmermann e colaboradores. ${ }^{15-}$ 17 Durante a execução do projeto, foram avaliadas a viabilidade econômica, segurança e reprodutibilidade do processo, visando a obtenção do mesilato de imatinibe dentro das especificações analíticas, num processo que possa ser reproduzido em escala industrial.

\section{Resultados e Discussão}

A síntese original do mesilato de imatinibe está descrita em duas patentes. A primeira descreve a obtenção da base livre imatinibe, ${ }^{15-17}$ e a segunda consiste na conversão desta base para o sal mesilato. ${ }^{30} \mathrm{~A}$ base livre imatinibe pertence à classe dos derivados FAP, conhecidos pela atividade antineoplásica. Os FAP são preparados através de rotas clássicas para a obtenção do sistema pirimidínico. ${ }^{16,31,32}$ A síntese descrita por Zimmermann e colaboradores é composta por 6 etapas e apresenta algumas desvantagens (Figura 3).

As principais desvantagens da síntese descrita por Zimmermann consistem no uso de reagentes de difícil manipulação e na necessidade de três etapas de reação para a obtenção da enaminona 5. Além disso, a referência original não descreveu os rendimentos das etapas de reação e não mostrou a estratégia sintética para a conversão da base livre (11) para o sal de imatinibe (3). ${ }^{15-17}$

Este trabalho, apesar de estar baseado em uma metodologia já descrita na literatura, apresenta muitas modificações que melhoraram o processo original, como as que serão mostradas a seguir (Figura 4).

A primeira etapa consistiu na síntese da enaminona 5 através da reação entre a 3acetilpiridina (4) e $\mathrm{N}, \mathrm{N}$-dimetilformamidadimetilacetal, levando ao produto em rendimento de $77 \%$. Entretanto, a obtenção de 5, por esta metodologia, exigiu um elevado tempo de reação. Uma etapa paralela levou a obtenção do intermediário 2metil-5-nitro-fenilguanidina (7) com o uso de cianamida, na presença de ácido nítrico. Esta etapa é considerada como limitante no processo devido ao baixo rendimento observado. No entanto, o diferencial desta etapa em relação ao procedimento original, está no isolamento do produto obtido. Foi realizada uma filtração do produto bruto com acetato de etila quente, levando à obtenção do intermediário 7, puro, em $37 \%$ de rendimento. Com isto, observou-se a total recuperação do material de partida não reagido, que pode ser reutilizado em uma nova reação. A obtenção do composto 8 foi realizada através da reação entre os compostos 5 e 7. Esta reação foi conduzida na presença de isopropanol e hidróxido de sódio $(\mathrm{NaOH})$, à temperatura de $80{ }^{\circ} \mathrm{C}$, por 15 horas, levando à obtenção do produto puro com rendimento de $89 \%$ (Figura 4). 


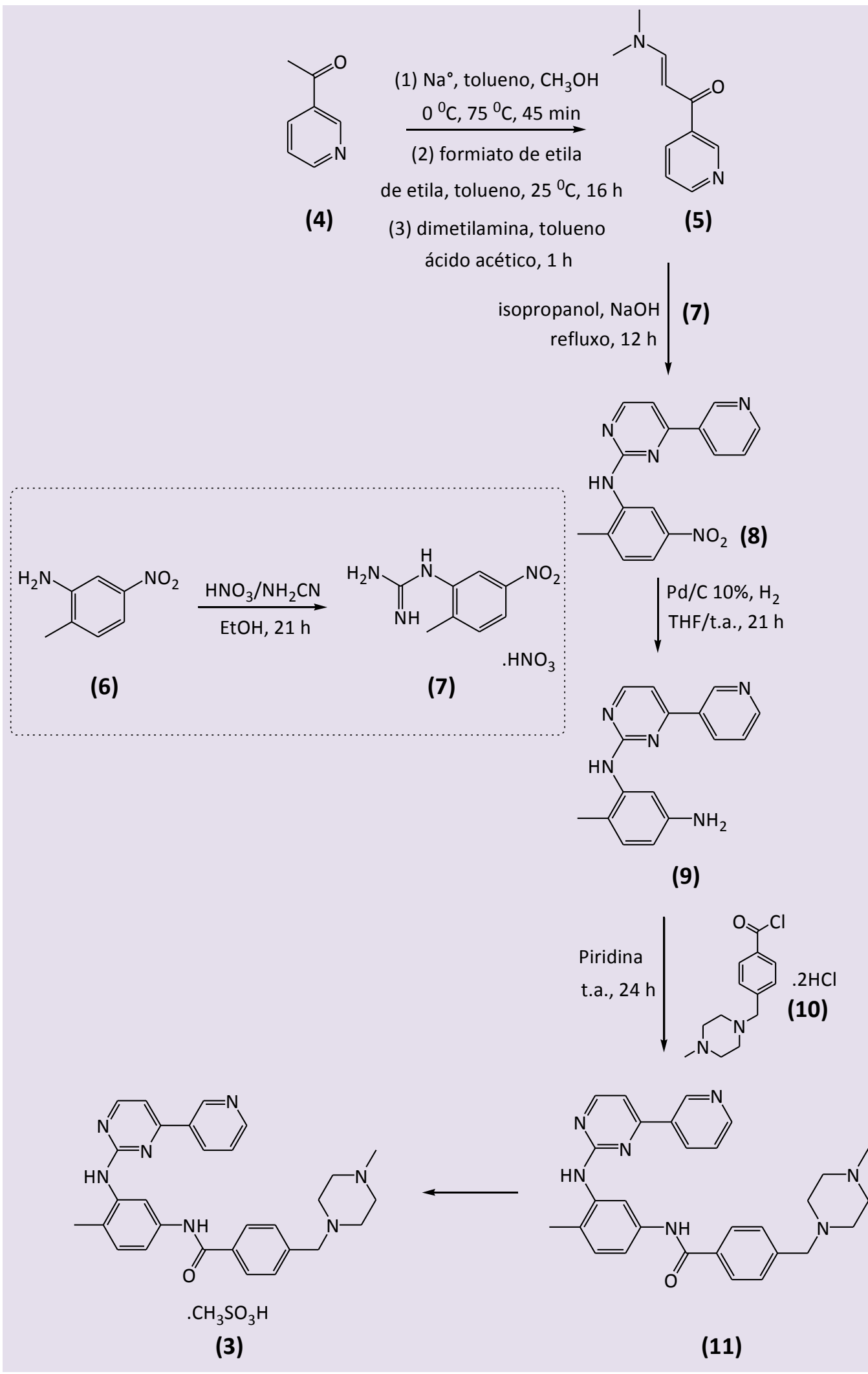

Figura 3. Esquema da rota sintética desenvolvida por Zimmermann e colaboradores

A redução do grupo nitro do intermediário 8 é descrita na literatura utilizando-se tetrahidrofurano e paládio sobre carvão (THF e
$\mathrm{Pd} / \mathrm{C}$ 10\%). ${ }^{15-17}$ A suspensão foi agitada, durante vinte e uma horas, à temperatura ambiente, sob atmosfera de hidrogênio, 
levando à formação do produto com rendimento de $69 \%$. Na tentativa de melhorar o rendimento desta etapa e empregar um catalisador mais barato, utilizou-se ferro como catalisador. Desta forma, a obtenção do $\mathrm{N}$-(5-amino-2-metilfenil)-4-(3-piridil)-2-pirimidinamina (9) foi realizada utilizando o sistema ferro/cloreto de amônio $\left(\mathrm{Fe} / \mathrm{NH}_{4} \mathrm{Cl}\right)$, em etanol/água. $\mathrm{O}$ meio de reação foi agitado à temperatura de $80{ }^{\circ} \mathrm{C}$, por 17 horas, resultando na formação de 9 , em $89 \%$ de rendimento (Figura 4).

Para obtenção do intermediário 10 , iniciou-se uma rota paralela através da reação entre o ácido 4-(clorometil)-benzoico (12) e a 1-metilpiperazina (13), ocorrendo formação do intermediário 14 com rendimento de $83 \% .{ }^{30}$ Posteriormente, o ácido 14 foi convertido ao correspondente cloreto de ácido 10 na forma de dicloridrato, em $92 \%$ de rendimento, empregando cloreto de tionila (Figura 4). Vale ressaltar que este intermediário é obtido e mantido na forma de sal para a etapa posterior, devido à sua maior estabilidade e facilidade de manipulação.

A síntese convergente para a obtenção do imatinibe (11) tem como última etapa a condensação entre os compostos 9 e $\mathbf{1 0}$. A literatura descreve o uso da piridina como solvente e aceptor de $\mathrm{H}^{+} ; i^{15-17}$ porém, os ensaios realizados utilizando esta metodologia apresentaram rendimentos na faixa de $10 \%$. Desta forma, foi necessária a utilização de outro método para a obtenção de 11. Na reação entre os intermediários 9 e 10 foi utilizado como aceptor o 4,4,- dimetilamino piridina (DMAP), em diclorometano à temperatura ambiente, por 48 horas, obtendo-se a base livre com rendimento de $41 \%$ (Figura 4).

A obtenção do mesilato de imatinibe (3), princípio ativo do glivec $^{\circledR}$, consistiu, inicialmente, na reação entre o intermediário 11 e o ácido metanossulfônico na presença de carvão ativo e metanol, à temperatura ambiente. Porém, houve perdas significativas com esta metodologia devido à solubilidade parcial do sal formado em metanol, que provocou uma pequena precipitação do produto final no meio. Desta forma, buscouse outro método para a obtenção deste composto (Figura 4).

O mesilato de imatinibe (3) é um sal insolúvel em acetona; por conseguinte, a sua base é parcialmente solúvel. Partindo-se destas características foi realizada uma recristalização em acetona, com tratamento prévio da base livre em carvão ativo, obtendo-se um líquido amarelo claro. Sobre esta solução, resfriada em banho de gelo, foi gotejada uma solução de ácido metanossulfônico, levando à precipitação do mesilato de imatinibe (3) com rendimento final de $71 \%$ (Figura 4).

Todos os experimentos para a obtenção deste produto foram realizados em escala semi-piloto. Vale ressaltar que os rendimentos descritos referem-se aos intermediários e ao produto final puro. Os processos de purificação empregados em cada etapa, quando necessários, estão descritos na seção experimental. 
<smiles>CC(=O)c1cccnc1</smiles>

(4)

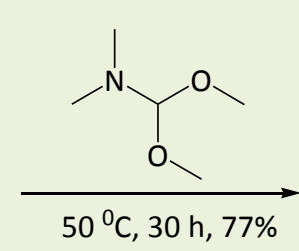<smiles>CN(C)CCC(=O)c1cccnc1</smiles>

(5)

(7) $\mid \begin{aligned} & \text { isopropanol, } \mathrm{NaOH} \\ & 80^{\circ} \mathrm{C}, 30 \mathrm{~h}, 77 \%\end{aligned}$

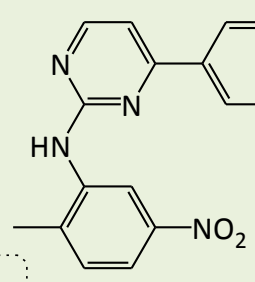

(8)

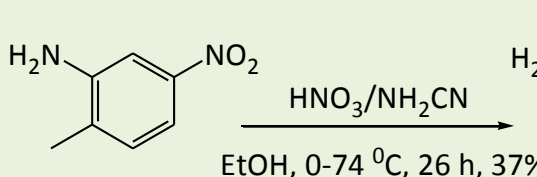

(6)<smiles>Cc1ccc([N+](=O)[O-])cc1NC(=N)N</smiles>

(7)

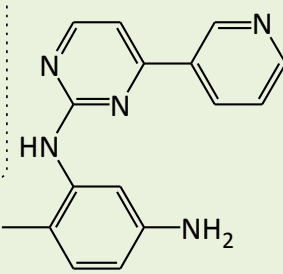

$\mathrm{Fe} / \mathrm{NH}_{4} \mathrm{Cl}$ $\mathrm{H}_{2} \mathrm{O} / \mathrm{EtOH}, 80^{\circ} \mathrm{C}, 17 \mathrm{~h}, 89 \%$

(9)

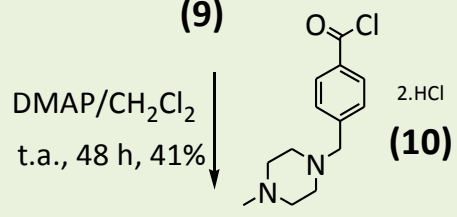

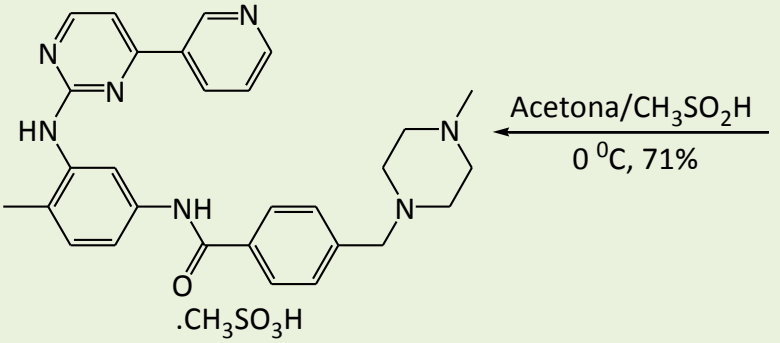

(3)

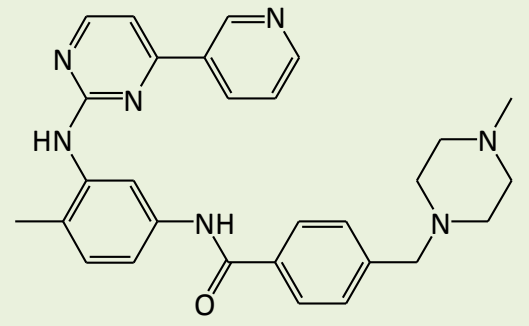

(11)

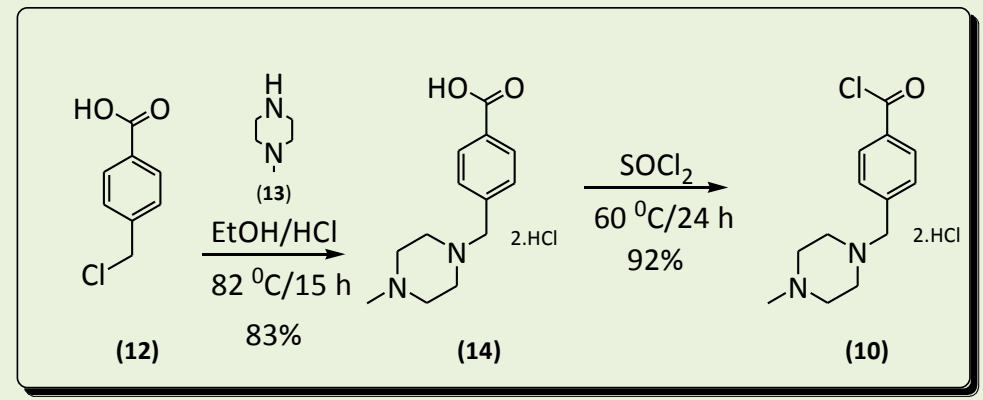

Figura 4. Rota sintética desenvolvida para a obtenção do mesilato de imatinibe 3 


\section{Conclusão}

A verticalização da produção de medicamentos para o Brasil é ressaltada em foros de ciência e tecnologia mostrando a importância da produção nacional com capacitação de mão-de-obra, desenvolvimento de know how e obtenção de tecnologia necessária para responder às demandas farmacêuticas instaladas no país. Neste âmbito, o governo apoia as iniciativas que possam contribuir para um país mais autossuficiente. Além disso, as PDPs entre os laboratórios públicos e o MS são beneficiadas pelo desenvolvimento de produtos. Assim, o desenvolvimento da síntese do glivec $^{\circledR}$ pode contribuir diretamente para o fortalecimento deste segmento no país.

Após a avaliação experimental do processo de síntese do mesilato de imatinibe disponível na literatura, foi desenvolvida uma metodologia sintética capaz de produzir o sal de imatinibe (3) com rendimento global de $5 \%$ e dentro das especificações descritas na literatura.

Após a obtenção do produto, pode-se observar que o processo, apesar de descrito na literatura, foi otimizado, visando tornar-se uma metodologia factível para ser empregada na produção industrial brasileira.

Nota: Em 2011, este trabalho recebeu o prêmio de Reconhecimento Técnico de Inovação no 5o ENIFarMed, Instituto de Pesquisa e Desenvolvimento de Fármacos e Produtos Farmacêuticos, IPD Farma.

\section{Experimental}

\subsection{Equipamentos, reagentes e materiais}

Para a determinação dos pontos de fusão foi utilizado um aparelho Büchi B-545 e os valores foram corrigidos. Os espectros de infravermelho (IV) foram obtidos em espectrofotômetro Nicolet-205 sobre brometo de potássio $(\mathrm{KBr})$. Os valores para as absorções foram referidos em número de onda, utilizando-se como unidade o centímetro recíproco $\left(\mathrm{cm}^{-1}\right)$. Os espectros de ressonância magnética nuclear (RMN) foram obtidos em um aparelho Bruker HC $200 \mathrm{MHz}$. Os deslocamentos químicos $(\delta)$ foram expressos em partes por milhão (ppm) e os valores das constantes de acoplamento $(J)$ em Hertz $(\mathrm{Hz})$.

As análises de HPLC analítico foram feitas em aparelho Shimadzu com duas bombas modelo LC-10AD, autoinjetor Rhedyne modelo SIL-10AD com loop de $20 \mu \mathrm{L}$ e detector por varredura de absorção de UV modelo SPD-M10A. As corridas cromatográficas foram acompanhadas simultaneamente em 215, 267 e $285 \mathrm{~nm}$ com varredura de espectro entre $190-370 \mathrm{~nm}$. Em todas as análises, o fluxo da fase móvel foi de $1,2 \mathrm{~mL} / \mathrm{min}$, sendo o volume injetado correspondente ao loop completo. Nos ensaios cromatográficos com fase reversa foi empregada coluna Symmetry $C_{18}, 4,6 \times 250$ $\mathrm{nm}$, com fase de sílica com $5 \mu \mathrm{m}$ de diâmetro de partículas esféricas.

Os espectros de massas foram obtidos em um sistema de espectrometria de massas por impacto de elétrons a $70 \mathrm{eV}$, com prévia separação por cromatografia gasosa (CG-EM) no aparelho HP6890. Foi utilizada uma coluna com fase SE54, com $30 \mathrm{~cm}$ de comprimento, $0,25 \mu$ de espessura do filme e $0,25 \mathrm{~mm}$ de diâmetro interno. $O$ gás carreador foi o hélio, ao fluxo de $1 \mathrm{~mL} / \mathrm{min}$ e split de 1:20.

A espectrometria de massas com ionização por eletrospray em modo negativo (ESI-EM) foi realizada em equipamento Waters ${ }^{\circledR}$ Micromass ZQ4000 nas condições de 2,80-4,00 kV (voltagem capilar) e 15-50 V (voltagem do cone). Os valores obtidos estão expressos em relação massa/carga $(\mathrm{m} / \mathrm{z})$ e são equivalentes à massa molecular da substância menos um próton.

Os reagentes e materiais foram adquiridos comercialmente e utilizados sem purificação prévia. 
4.2. Obtenção do 3-dimetilamino-1-(3piridil)-2-propen-1-ona (5)

Em um reator de $1 \mathrm{~L}$ acoplado com agitador mecânico, condensador de refluxo e termômetro, foram adicionados 3acetilpiridina (4) $(50 \mathrm{~mL})(0,454 \mathrm{~mol})$ e $\mathrm{N}, \mathrm{N}$ dimetilformamida dimetil acetal $(67 \mathrm{~mL})$ $(0,500 \mathrm{~mol})$. O meio de reação foi mantido por agitação mecânica à temperatura de 50 o $C$ por 30 horas. O solvente formado na reação (metanol) foi retirado por destilação, obtendo-se um óleo escuro. A este óleo foram adicionados $60 \mathrm{~mL}$ de hexano, e a solução obtida foi deixada no freezer durante uma noite, o que levou à cristalização do produto. Os cristais, juntamente com o hexano, foram transferidos para um Erlenmeyer de $500 \mathrm{~mL}$ equipado com um agitador magnético. Foram adicionados 150 $\mathrm{mL}$ de tolueno e a mistura foi levemente aquecida até a completa solubilização. Adicionou-se 2,0 g de carvão ativado e, em seguida, filtrou-se a pressão normal. A este líquido resultante, adicionou-se $150 \mathrm{~mL}$ de hexano e deixou-se no freezer por uma noite. Os cristais amarelo-claros obtidos foram filtrados a vácuo e caracterizados como 3dimetilamino-1-(3-piridil)-2-propen-1-ona (5).

Rendimento: $77 \%$

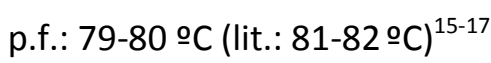

IV $\left(\mathrm{KBr}, \mathrm{v}, \mathrm{cm}^{-1}\right): 2925 ; 1643 ; 1587 ; 1538$.

CG-EM (70 eV), m/z (\%): 176 (12); 159 (35); 134 (10); 106 (23); 98 (91); 78 (50); 51 (100).

RMN ${ }^{1} \mathrm{H}\left(200 \mathrm{MHz}\right.$, DMSO- $\left.\mathrm{d}_{6}, \mathrm{TMS}, \delta\right)$ : 2,91 (s, 3H, $\left.\mathrm{CH}_{3}\right) ; 3,13$ (s, 3H, $\left.\mathrm{CH}_{3}\right) ; 5,83$ (d, $1 \mathrm{H}, \mathrm{J}=12 \mathrm{~Hz}, \mathrm{COC} \underline{\mathrm{H}}=\mathrm{CH}) ; 7,40-7,47(\mathrm{~m}, 1 \mathrm{H}, \mathrm{H}-$ 5); $7,75(\mathrm{~d}, 1 \mathrm{H}, J=12 \mathrm{~Hz}, \mathrm{COCH}=\mathrm{C} \underline{\mathrm{H}}) ; 8,18-$ $8,22(\mathrm{~m}, 1 \mathrm{H}, \mathrm{H}-4) ; 8,63(\mathrm{~d}, 1 \mathrm{H}, J=6 \mathrm{~Hz}, \mathrm{H}-6)$; $9,05$ (d, $1 \mathrm{H}, J=2 \mathrm{~Hz}, \mathrm{H}-2)$.

RMN ${ }^{13} \mathrm{C}\left(50 \mathrm{MHz}\right.$, DMSO-d $\left.\mathrm{d}_{6}, \delta\right): 37,2$ $\left(\mathrm{CH}_{3}\right) ; 44,5\left(\mathrm{CH}_{3}\right) ; 90,9(\mathrm{CO} \underline{\mathrm{CH}}=\mathrm{CH}) ; 123,4(\mathrm{C}-$ 3); 134,6 (C-4); 135,1 (C-5); 148,4 (C-6); 151,2 $(\mathrm{C}-2) ; 154,6(\mathrm{COCH}=\underline{\mathrm{CH}}) ; 184,0(\mathrm{C}=\mathrm{O})$.
4.3. Obtenção do nitrato de 2-metil-5nitro-fenilguanidina (7)

Em um reator de $1 \mathrm{~L}$ acoplado com agitador mecânico, condensador de refluxo e termômetro foram adicionados 2-metil-5nitroanilina (6) (50g) (0,328 mol) e álcool etílico P.A. (125 mL). A suspensão resultante foi agitada, em banho de gelo, e $23 \mathrm{~mL}(0,514$ mol) de ácido nítrico foram gotejados. Após a adição, o banho de gelo foi retirado e a suspensão foi aquecida, com agitação mecânica, à temperatura interna de 74 으, por 26 horas. Durante a elevação da temperatura, adicionou-se um total de $21 \mathrm{~mL}$ $(0,540 \mathrm{~mol})$ de solução de cianamida $50 \% \mathrm{v} / \mathrm{v}$ ( $3 \times 7 \mathrm{~mL}$ num período de 9 horas). Após o término do aquecimento, a solução castanha avermelhada foi transferida para um Erlenmeyer e deixada no freezer por uma noite. Os cristais amarelos foram filtrados por gravidade. Em seguida, foram lavados com $670 \mathrm{~mL}$ de acetato de etila aquecido para retirar o material de partida que não reagiu. Após a lavagem, o sólido foi seco em estufa a vácuo, à temperatura de 50 으, por 24 horas.

Rendimento: $37 \%$

p.f.: 214-216 ㅇ (lit.: 219-226 ㅇ) $)^{15-17}$

IV $\left(\mathrm{KBr}, \mathrm{cm}^{-1}\right): 3474 ; 3149 ; 1698 ; 1622 ;$ $1517 ; 1348$.

ESI-EM (3,93 kV; 21 V), m/z (\%): 195 (100).

RMN ${ }^{1} \mathrm{H}\left(200 \mathrm{MHz}\right.$, DMSO-d, $\mathrm{d}_{6}$ TMS, $\left.\delta\right)$ : 2,32 (s, 3H, $\left.\mathrm{CH}_{3}\right) ; 7,46\left(\mathrm{~s}, 3 \mathrm{H}, \mathrm{NH}_{2}, \mathrm{NH}\right) ; 7,63$ (d, $1 \mathrm{H}, \mathrm{J}=10 \mathrm{~Hz}, \mathrm{H}-6) ; 8,08-8,16(\mathrm{~m}, 2 \mathrm{H}, \mathrm{H}-3 \mathrm{e}$ $\mathrm{H}-4)$; 9,54 (s, 1H, NH).

RMN ${ }^{13} \mathrm{C}(50 \mathrm{MHz}$, DMSO-d $6, \delta): 17,5$ $\left(\mathrm{CH}_{3}\right) ; 122,5$ (C-6); 122,7 (C-4); 132,2 (C-3); 134,5 (C-2); 143,6 (C-1); 146,3 (C-5); 156,2 ( $\mathrm{C}=\mathrm{NH})$.

\subsection{Obtenção do $N$-(2-metil-5-nitrofenil)-} 4-(3-piridil)-2-pirimidinamina (8)

Em um reator de $1 \mathrm{~L}$ acoplado com agitador mecânico, condensador de refluxo e termômetro, foram adicionados 2-metil-5nitro-fenilguanidina (7) $(24,82 \mathrm{~g})(0,103 \mathrm{~mol})$, 3-dimetilamino-1-(3-piridil)-2-propen-1-ona (5) (17 g) (0,096 mol), isopropanol (200 mL) e 
$\mathrm{NaOH}(4,25$ g) $(0,106$ mol). A suspensão avermelhada resultante foi aquecida, com agitação mecânica, à temperatura interna de

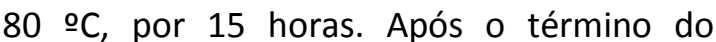
aquecimento, a solução castanha foi transferida para um Erlenmeyer e deixada no freezer por uma noite. O sólido marrom resultante foi filtrado a vácuo e seco em estufa a vácuo, à temperatura de $60 \stackrel{\circ}{ } \mathrm{C}$, por 24 horas.

Rendimento: $89 \%$

p.f.: $193-195$ ㅇ (lit.: 195-198 ㅇ C $)^{15-17}$

IV $\left(\mathrm{KBr}, \mathrm{cm}^{-1}\right): 3247 ; 1583 ; 1535 ; 1382$.

ESI-EM (2,80 Kv; 25 V), m/z (\%): 308 (55).

RMN ${ }^{1} \mathrm{H}\left(200 \mathrm{MHz}\right.$, DMSO-d $\left.\mathrm{d}_{6}, \mathrm{TMS}, \delta\right):$ 2,41 (s, 3H, $\left.\mathrm{CH}_{3}\right) ; 7,48-7,57$ (m, 3H, H-3 fenil, $\mathrm{H}-5$ pirimidina, $\mathrm{H}-5$ piridina); $7,87(\mathrm{dd}, 1 \mathrm{H}, \mathrm{J}=$ $8 \mathrm{~Hz}$ e $2 \mathrm{~Hz}, \mathrm{H}-4$ fenil); 8,46 (dt, $1 \mathrm{H}, J=8 \mathrm{~Hz}$ e $2 \mathrm{~Hz}, \mathrm{H}-4$ piridina); $8,60(\mathrm{~d}, 1 \mathrm{H}, \mathrm{J}=6 \mathrm{~Hz}, \mathrm{H}-6$ pirimidina); 8,70 (dd, $1 \mathrm{H}, J=6 \mathrm{~Hz}$ e $2 \mathrm{~Hz}, \mathrm{H}-6$ piridina); 8,79 (d, $1 \mathrm{H}, J=2 \mathrm{~Hz}, \mathrm{H}-6$ fenil); 9,19 (s, $1 \mathrm{H}, \mathrm{NH}$ ); 9,30 (d, $1 \mathrm{H}, J=2 \mathrm{~Hz}, \mathrm{H}-2$ piridina).

RMN ${ }^{13} \mathrm{C}\left(50 \mathrm{MHz}\right.$, DMSO-d $\left.\mathrm{d}_{6}, \delta\right): 18,3$ $\left(\mathrm{CH}_{3}\right) ; 108,8$ (C-6 fenil); 117,4 (C-5 pirimidina); 117,9 (C-4 fenil); 123,8 (C-2 fenil); 131,2 (C-5 piridina); 131,8 (C-3 fenil); 134,2 (C-4 piridina); 138,6 (C-3 piridina); 138,8 (C-5 fenil); 145,8 (C-2 piridina); 148,1 (C-6 piridina); 151,6 (C-6 pirimidina); 159,6 (C-1 fenil); 160,2 (C-4 pirimidina); 161,5 (C-2 pirimidina).

4.5. Obtenção do $N$-(5-amino-2-metilfenil)-4-(3-piridil)-2-pirimidinamina (9)

Em um reator de $1 \mathrm{~L}$ acoplado com agitador mecânico, condensador de refluxo e termômetro, foram adicionados $\mathrm{N}$-(2-metil-5nitrofenil)-4-(3-piridil)-2-pirimidinamina (21 g) $(0,068 \mathrm{~mol})$ e uma mistura de água $(416 \mathrm{~mL})$ e álcool etílico $(218 \mathrm{~mL})$, cloreto de amônio $(3,7$ g) $(0,069 \mathrm{~mol})$ e ferro metálico $(21,5 \mathrm{~g})(0,384 \mathrm{~mol})$. A suspensão resultante foi aquecida à temperatura interna de $80 \stackrel{\circ}{\circ}$, com agitação mecânica, por 17 horas. Após o término do aquecimento, a solução castanha foi filtrada sob celite e o solvente foi removido à pressão reduzida, resultando em um óleo castanho que, após secagem em estufa a vácuo, sem aquecimento, por 24 horas, formou um sólido castanho escuro.

Rendimento: 89\%

p.f.: $133-135$ ㅇ (lit.: 138-140 ㄷ) $)^{15-17}$

IV $\left(\mathrm{KBr}, \mathrm{cm}^{-1}\right): 3421 ; 1579 ; 1554 ; 1449$; $1415 ; 800$.

ESI-EM (2,80 Kv; 25 V), m/z (\%): 278 (70).

RMN ${ }^{1} \mathrm{H}\left(200 \mathrm{MHz}\right.$, DMSO-d $\left.\mathrm{d}_{6}, \mathrm{TMS}, \delta\right)$ : $2,00\left(\mathrm{~s}, 3 \mathrm{H}, \mathrm{CH}_{3}\right) ; 6,34(\mathrm{~d}, 1 \mathrm{H}, J=8 \mathrm{~Hz}, \mathrm{H}-4$ fenil); $6,46(\mathrm{~d}, 1 \mathrm{H}, J=8 \mathrm{~Hz}, \mathrm{H}-6$ fenil); 6,76$6,93\left(\mathrm{~m}, 3 \mathrm{H}, \mathrm{NH}_{2}, \mathrm{H}-3\right.$ fenil); 7,33 (d, $1 \mathrm{H}, J=4$ $\mathrm{Hz}, \mathrm{H}-5$ pirimidina); 7,50 (d, $1 \mathrm{H}, J=4 \mathrm{~Hz}, \mathrm{H}-5$ piridina); $8,32-8,44(\mathrm{~m}, 2 \mathrm{H}, \mathrm{H}-4$ piridina e $\mathrm{H}-6$ pirimidina); $8,62-8,67(\mathrm{~m}, 2 \mathrm{H}, \mathrm{H}-6$ piridina e $\mathrm{H}-2$ piridina); $9,20-9,21(\mathrm{~s}, 1 \mathrm{H}, \mathrm{NH})$.

RMN ${ }^{13} \mathrm{C}\left(50 \mathrm{MHz}\right.$, DMSO-d $\left.\mathrm{d}_{6}, \delta\right): 16,3$ $\left(\mathrm{CH}_{3}\right) ; 107,1$ (C-6 fenil); 111,1 (C-5 pirimidina); 112,3 (C-4 fenil); 113,8 (C-2 fenil); 120,8 (C-5 piridina); 123,8 (C-3 fenil); 130,3 (C-3 piridina); 131,3 (C-4 piridina); 134,3 (C-1 fenil); 137,9 (C-5 fenil); 146,8 (C-2 piridina); 147,8 (C-6 piridina); 156,4 (C-6 pirimidina); 158,8 (C-4 pirimidina); 161,5 (C-2 pirimidina).

\subsection{Obtenção do dicloroidrato do ácido 4-(4-metilpiperazinometil)benzóico (14)}

Em um reator de $1 \mathrm{~L}$ acoplado com agitador mecânico, condensador de refluxo e termômetro, foram adicionados o ácido 4(clorometil)-benzoico (12) (31,2 g) (0,183 mol), álcool etílico $(274 \mathrm{~mL})$ e uma solução de 1-metilpiperazina (13) $(89,5 \mathrm{~mL})(0,804 \mathrm{~mol})$ em álcool etílico $(91 \mathrm{~mL})$. A solução resultante foi aquecida, com agitação mecânica, à temperatura interna de 82 으, por 15 horas. Após este período, o álcool etílico foi retirado do meio de reação através de evaporação à pressão reduzida. $O$ produto obtido foi resfriado em banho de gelo e transferido em seguida para um funil de separação de $1 \mathrm{~L}$. Foi adicionado uma solução aquosa de $\mathrm{NaOH} 3 \mathrm{~N}$ (182 $\mathrm{mL}$ ) e a fase aquosa 
foi extraída com éter etílico ( $3 \times 182 \mathrm{~mL}$ ). A fase aquosa foi vertida para um reator encamisado de $1 \mathrm{~L}$, com temperatura ajustada para $-5 \stackrel{\circ}{\circ}$, acoplado com agitador mecânico e termômetro. Foi adicionado $\mathrm{HCl}$ concentrado $(150 \mathrm{~mL})(4,89 \mathrm{~mol})$, gota a gota, até atingir $\mathrm{pH} 2$, evitando-se a elevação da temperatura interna. Ocorreu precipitação do produto desejado, e este foi filtrado a vácuo e lavado com $70 \mathrm{~mL}$ de álcool etílico gelado. $O$ sólido bege obtido foi seco em estufa a vácuo, à temperatura de 80 으, por 24 horas.

Rendimento: $83 \%$

p.f.: $319-322$ ㅇ (lit.: 310-312 으)

IV $\left(\mathrm{KBr}, \mathrm{cm}^{-1}\right): 3012 ; 2985 ; 1714 ; 1461$; $1419 ; 1232$.

ESI-EM (3,93 Kv; 21 V), m/z (\%): 235 (100).

RMN ${ }^{1} \mathrm{H}\left(200 \mathrm{MHz}\right.$, DMSO-d $\left.\mathrm{d}_{6}, \mathrm{TMS}, \delta\right)$ : $2,79\left(\mathrm{~s}, 3 \mathrm{H}, \mathrm{CH}_{3}\right) ; 3,18-3,55\left(\mathrm{~m}, 8 \mathrm{H}, \mathrm{CH}_{2}\right.$ piperazina); 4,42 (s, $\left.2 \mathrm{H}, \mathrm{C}-\mathrm{CH}_{2}-\mathrm{N}\right) ; 7,69-7,78$ ( $m, 2 \mathrm{H}, \mathrm{H}-3$ e H-5 Ar); 7,95-8,02 (m, 2H, H-2 e $\mathrm{H}-6 \mathrm{Ar}) ; 12,0(\mathrm{~s}, 1 \mathrm{H}, \mathrm{OH})$.

RMN ${ }^{13} \mathrm{C}\left(50 \mathrm{MHz}\right.$, DMSO-d $\left.\mathrm{d}_{6}, \delta\right): 41,7$ $\left(\mathrm{CH}_{3}\right) ; 47,5$ (C-3 e C-5 piperazina); 49,5 (C-2 e C-6 piperazina); $58,0\left(\mathrm{C}-\mathrm{CH}_{2}-\mathrm{N}\right) ; 129,6(\mathrm{C}-3 \mathrm{e}$ C-5 Ar); 131,2 (C-2 e C-6 Ar); 131,5 (C-1 Ar); 135,3 (C-4 Ar); 166,9 (C=0).

\subsection{Obtenção do dicloroidratado do cloreto do ácido 4-(4-metilpiperazinometil) benzoico (10)}

Em um reator de $1 \mathrm{~L}$ acoplado com agitador mecânico, condensador de refluxo e termômetro, foram adicionados o dicloridrato do ácido 4-(4metilpiperazinometil) benzoico (14) (22 g) $(0,072 \mathrm{~mol})$ e o cloreto de tionila $(550 \mathrm{~mL})$ $(7,57 \mathrm{~mol})$. A suspensão resultante foi aquecida, com agitação mecânica, à temperatura interna de $60 \stackrel{\circ}{ } \mathrm{C}$, por 24 horas. Após o término do aquecimento, foram adicionados $500 \mathrm{~mL}$ de tolueno e a

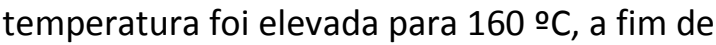
que houvesse a destilação do tolueno juntamente com o excesso de cloreto de tionila. Após a destilação, adicionou-se mais $200 \mathrm{~mL}$ de tolueno e repetiu-se o mesmo procedimento. A suspensão resultante foi colocada em banho de gelo e o sólido formado foi filtrado a vácuo, sendo imediatamente seco em estufa a vácuo, à temperatura de $100 \stackrel{\circ}{ } \mathrm{C}$, por 24 horas.

Rendimento: 92\%

$$
\text { p.f.: } 276-278 \stackrel{\circ}{\circ} \text { (lit.: 260-263 ㅇ) })^{30}
$$

IV $\left(\mathrm{KBr}, \mathrm{cm}^{-1}\right): 2977 ; 1714 ; 1419 ; 1232$.

RMN ${ }^{1} \mathrm{H}\left(200 \mathrm{MHz}\right.$, DMSO-d $\left.\mathrm{d}_{6}, \mathrm{TMS}, \delta\right)$ : 2,79 (s, 3H, $\left.\mathrm{CH}_{3}\right) ; 3,48-3,56(\mathrm{~m}, 8 \mathrm{H}, \mathrm{H}-2, \mathrm{H}-3$, $\mathrm{H}-5$ e H-6 piperazina); 4,43 (s, $\left.2 \mathrm{H}, \mathrm{C}-\mathrm{CH}_{2}-\mathrm{N}\right)$; 7,77 (d, $2 \mathrm{H}, \mathrm{J}=8 \mathrm{~Hz}, \mathrm{H}-3$ e H-5 Ar); 7,97 (d, 2H, $J=8 \mathrm{~Hz}, \mathrm{H}-2$ e H-6).

RMN ${ }^{13} \mathrm{C}\left(50 \mathrm{MHz}\right.$, DMSO-d $\left.\mathrm{d}_{6}, \delta\right): 47,4$ $\left(\mathrm{CH}_{3}\right) ; \quad 49,0 \quad\left(\mathrm{C}-\mathrm{CH}_{2}-\mathrm{N}\right) ; \quad 57,5 \quad(\mathrm{C}-3$ e $\mathrm{C}-5$ piperazina); 57,7 (C-2 e C-6 piperazina); 129,5 (C-2 e C-6 Ar); 131,4 (C-3 e C-5 Ar); 133,5 (C-1 $\mathrm{Ar}) ; 134,5$ (C-4 Ar); 166,8 (C=0).

(11)

\subsection{Obtenção do imatinibe base livre}

Em um reator de $1 \mathrm{~L}$ acoplado com agitador mecânico, foram adicionados o dicloridrato do cloreto do ácido 4-(4metilpiperazinometil) benzoico (10) $(19,2 \mathrm{~g})$ (0,059 mol), $N$-(5-amino-2-metil-fenil)-4-(3piridil)-2-pirimidinamina (9) (22 g) (0,079 mol), 4,4-dimetilaminopiridina (14,5 g) $(0,118$ mol) e diclorometano $(580 \mathrm{~mL})$. A suspensão resultante foi agitada por 48 horas. 0 solvente foi removido à pressão reduzida, em evaporador. O óleo escuro resultante foi lavado com água $(10 \times 100 \mathrm{~mL})$ e a purificação foi feita através de um coluna de Sephadex LH20, utilizando metanol puro (5 L) como eluente.

Rendimento: $41 \%$

p.f.: 198-200 ㅇ (lit.: 211-213 ㄷ) $)^{15-17}$

IV $\left(\mathrm{KBr}, \mathrm{cm}^{-1}\right): 3420 ; 3278 ; 1648 ; 1576$; $1529 ; 1451 ; 1416 ; 1290$.

ESI-EM (3,61 Kv; 50 V), m/z (\%): 493 (100). 
$\mathrm{RMN}^{1} \mathrm{H}\left(200 \mathrm{MHz}, \mathrm{DMSO}-\mathrm{d}_{6}, \mathrm{TMS}, \delta\right): 2,07$ (s, $\left.3 \mathrm{H}, \mathrm{CH}_{3}\right) ; 2,21\left(\mathrm{~s}, 3 \mathrm{H}, \mathrm{CH}_{3}\right) ; 2,57\left(\mathrm{~s}, 8 \mathrm{H}, \mathrm{CH}_{2}\right.$ piperazina); 3,61 (s, 2H, C- $\left.\mathrm{CH}_{2}-\mathrm{N}\right) ; 7,19(\mathrm{~d}, 1 \mathrm{H}$, $\mathrm{J}=10 \mathrm{~Hz}, \mathrm{H}-5$ pirimidina); 7,40-7,54 (m, 5H, H$2 \mathrm{Ar}, \mathrm{H}-5 \mathrm{Ar}, \mathrm{H}-6 \mathrm{Ar}$ e H-2 Ar e H-6 Ar); 7,93 (d, $1 \mathrm{H}, \mathrm{J}=8 \mathrm{~Hz}, \mathrm{H}-4$ piridina); 8,08 (s, $1 \mathrm{H}, \mathrm{H}-6$ piridina); $8,43-8,51$ ( $\mathrm{m}, 1 \mathrm{H}, \mathrm{H}-5$ piridina); 8,67 (d, $1 \mathrm{H}, J=10 \mathrm{~Hz}, \mathrm{H}-4$ pirimidina); $8,98(\mathrm{~s}, 1 \mathrm{H}$, $\mathrm{NH}) ; 9,26(\mathrm{~s}, 1 \mathrm{H}, \mathrm{NH}) ; 10,2(\mathrm{~s}, 1 \mathrm{H}, \mathrm{H}-2$ piridina).

RMN ${ }^{13} \mathrm{C}\left(50 \mathrm{MHz}\right.$, DMSO-d $\left.\mathrm{d}_{6}, \delta\right): 17,5$ $\left(\mathrm{CH}_{3}\right) ; 42,9\left(\mathrm{CH}_{3}\right) ; 49,9$ (C-3 e C-5 piperazina); 52,9 (C-2 e C-6 piperazina); 60,6 (C- $\left.\mathrm{CH}_{2}-\mathrm{N}\right)$; 107,4 (C-2 Ar); 116,7 (C-4 Ar); 117,2 (C-5 pirimidina); $123,7 \quad$ (C-6 Ar); 127,6 (C-5 piridina); 128,7 (C-3 e C-5 Ar); 129,9 (C-2 e C$6 \mathrm{Ar}$ ); 132,1 (C-5 Ar); 133,9 (C-4 Ar); 134,3 (C4 piridina); 137,1 (C-1 Ar); 137,7 (C-3 piridina); $\quad 141,0 \quad\left(\underline{\mathrm{C}}-\mathrm{CH}_{2}-\mathrm{N}\right) ; \quad 148,1 \quad$ (C-2 piridina); 150,1 (C-6 piridina); 151,3 (C-3 Ar); 159,4 (C-6 pirimidina); 161,1 (C-4 pirimidina); $161,5$ (C-2 pirimidina); 165,1 ( $C=0)$.

CLAE: 98\% em 25,9 minutos.

\subsection{Obtenção do mesilato de imatinibe} (3)

Em um reator de $1 \mathrm{~L}$ acoplado com agitador mecânico, foram adicionados o imatinibe base livre (11) (16 g) $(0,032 \mathrm{~mol})$ e acetona $(1 \mathrm{~L})$. Esta mistura foi aquecida até formar uma solução. Adicionou-se $8 \mathrm{~g}$ de carvão ativo e após 20 minutos de aquecimento, filtrou-se a solução à pressão normal. Este líquido resultante foi colocado em banho de gelo, e, em seguida, sobre ele foi gotejada uma solução contendo ácido metanossulfônico e acetona $(1: 1 \mathrm{v} / \mathrm{v})$, até a completa precipitação do produto $(\mathrm{pH} 3)$. Os cristais amarelo-claros obtidos foram filtrados à vácuo e secos em estufa a vácuo, à temperatura de $100{ }^{\circ} \mathrm{C}$, por 48 horas.

Rendimento: $71 \%$

p.f.: $212-213 \stackrel{\circ}{\circ}$ (lit.: $217 \stackrel{\circ}{ }$ C) ${ }^{15-17}$

IV $\left(\mathrm{KBr}, \mathrm{cm}^{-1}\right): 3322 ; 2792 ; 1648 ; 1583$; $1552 ; 1415 ; 1282 ; 800$.
RMN ${ }^{1} \mathrm{H}(200 \mathrm{MHz}$, DMSO-d $6, \mathrm{TMS}, \delta):$ $2,16\left(\mathrm{~s}, 3 \mathrm{H}, \mathrm{CH}_{3}\right) ; 2,21\left(\mathrm{~s}, 3 \mathrm{H}, \mathrm{CH}_{3}\right) ; 2,36(\mathrm{~s}$, $11 \mathrm{H}, \mathrm{CH}_{2}$ piperazina e $\mathrm{CH}_{3}$ mesilato); 3,51 (s, $\left.2 \mathrm{H}, \mathrm{C}-\mathrm{CH}_{2}-\mathrm{N}\right) ; 7,19(\mathrm{~d}, 1 \mathrm{H}, J=10 \mathrm{~Hz}, \mathrm{H}-5$ pirimidina); 7,40-7,54 (m, 5H, H-2 Ar, H-5 Ar, H-6 Ar e H-2 Ar e H-6 Ar); 7,89 (d, $1 \mathrm{H}, J=8 \mathrm{~Hz}$, $\mathrm{H}-4$ piridina); 8,08 (s, $1 \mathrm{H}, \mathrm{H}-6$ piridina); $8,44-$ $8,51(\mathrm{~m}, 1 \mathrm{H}, \mathrm{H}-5$ piridina); $8,67(\mathrm{~d}, 1 \mathrm{H}, \mathrm{J}=10$ $\mathrm{Hz}, \mathrm{H}-4$ pirimidina); 8,97 (s, $1 \mathrm{H}, \mathrm{NH}) ; 9,26$ (s, $1 \mathrm{H}, \mathrm{NH}) ; 10,1$ (s, $1 \mathrm{H}, \mathrm{H}-2$ piridina).

RMN ${ }^{13} \mathrm{C}\left(50 \mathrm{MHz}\right.$, DMSO-d $\left.\mathrm{d}_{6}, \delta\right): 17,5$ $\left(\mathrm{CH}_{3}\right) ; 41,9\left(\mathrm{CH}_{3}\right) ; 47,5$ (C-3 e C-5 piperazina); 49,4 (C-2 e C-6 piperazina); 57,8 (C- $\left.\mathrm{CH}_{2}-\mathrm{N}\right)$; 107,9 (C-2 Ar); 117,2 (C-4 Ar); 117,3 (C-5 pirimidina); 126,4 (C-6 Ar); 127,8 (C-5 piridina); 128,1 (C-3 e C-5 Ar); 130,2 (C-2 e C$6 \mathrm{Ar}$ ); 131,1 (C-5 Ar); 132,9 (C-4 Ar); 134,6 (C4 piridina); 135,8 (C-1 Ar); 137,0 (C-3 piridina); $137,2 \quad\left(\underline{\mathrm{C}}-\mathrm{CH}_{2}-\mathrm{N}\right) ; \quad 141,2 \quad$ (C-2 piridina); 142,7 (C-6 piridina); 145,4 (C-3 Ar); 159,3 (C-6 pirimidina); 159,6 (C-4 pirimidina); 160,6 (C-2 pirimidina); 164,7 (C=0).

CLAE: 99\% em 22,5 minutos.

\section{Referências Bibliográficas}

${ }^{1}$ Sítio do WHO Cancer Control Programme. Disponível em: <http://www.who.int $>$. Acesso em: 15 outubro 2012.

${ }^{2}$ Instituto Nacional de Câncer José Alencar Gomes da Silva. Coordenação Geral de Ações Estratégicas. Coordenação de Prevenção e Vigilância. Estimativa 2012: incidência de câncer no Brasil / Instituto Nacional de Câncer José Alencar Gomes da Silva - Rio de Janeiro: Inca, 2011, $118 \mathrm{p}$.

${ }^{3}$ Sítio do Ministério da Saúde. Painel de Indicadores do SUS, volume 1, 2006. Disponível em: <http://portal.saude.gov.br>. Acesso em: 15 outubro 2012.

${ }^{4}$ Druker, B. J.; Sawyers, C. L.; Kantarjian, H.; Resta, D. J.; Reese, S. F.; Ford, J. M.; Capdeville, R.; Talpaz, M. New Engl. J. Med. 2001, 344, 1038. [CrossRef] [PubMed]

${ }^{5}$ Rowley, J. D. Nature 1973, 243, 290. [CrossRef] [PubMed] 
${ }^{6}$ O' Dwyer, M. E.; Druker, B. J. Lancet Oncol. 2000, 1, 207. [CrossRef] [PubMed]

${ }^{7}$ Avila, C. M.; Romeiro, N. C. Rev. Virtual Quim. 2010, 2, 59; [Link] Silva, B. V.; Horta, B. A. C.; de Alencastro, R. B.; Pinto, A. C. Quim. Nova 2009, 32, 453. [CrossRef]

${ }^{8}$ Yaish, P.; Gazit, A.; Gilon, C.; Levitzki, A. Science 1988, 242, 933. [CrossRef] [PubMed]

${ }^{9}$ Druker, B. J.; Lydon, N. B. J. Clin. Invest. 2000, 105, 3. [CrossRef]

${ }^{10}$ Kalesh, K. A.; Sim, D. S. B.; Wang, J.; Liu, K.; Lin, Q.; Yao, S. Q. Chem. Commun. 2010, 46, 1118. [CrossRef] [PubMed]

${ }^{11}$ Kalesh, K. A.; Liu, K.; Yao, S. Q. Org. Biomol. Chem. 2009, 7, 5129. [CrossRef] [PubMed]

${ }^{12}$ Mahboobi, S.; Dove, S.; Sellmer, A.;

Winkler, M.; Eichhorn, E.; Pongratz, $\mathrm{H}_{\text {.; }}$ Ciossek, T.; Baer, T.; Maier, T.; Beckers, T. J. Med. Chem. 2009, 52, 2265. [CrossRef] [PubMed]

${ }^{13}$ Choi, H. G.; Sim, T.; Gray, N.; Zhou, W.; Chang, J. W.; Zhang, J.; Weisberg, E. PCT Int. Appl. WO 2010144909 2010. (CA 154:64811) ${ }^{14}$ Cohen, M. H.; Johnson, J. R.; Justice, R.; Pazdur, R. Oncologist 2012, 17, 992. [CrossRef] [PubMed]

${ }^{15}$ Zimmermann, J. EP Patent 564,409, 1993. (CA 120:107056)

${ }^{16}$ Zimmermann, J. U.S. US5,521,184 1996. (CA 125:114681)

${ }^{17}$ Zimmermann, J.; Buchdunger, E.; Mett, H.; Meyer, T.; Lydon, N. B. Bioorg. Med. Chem. Lett. 1997, 7, 187. [CrossRef]

${ }^{18}$ Potluri, R. B.; Hariharakrishnan, V. S.; Singh, A. N.; Raju, G. V. M.; Samy, M. L.; Prasad, K. H. Indian Pat. Appl. IN2007CH00006 2008. (CA 152:119644)

${ }^{19}$ Hopkin, M. D.; Baxendale, I. R.; Ley, S. V. Chem. Commun. 2010, 46, 2450. [CrossRef] [PubMed]
${ }^{20}$ Song, H.; Peng, X.; Lin, D.; Yang, M.; Sun, Z.; Ma, C.; Han, P. Faming Zhuanli Shenqing CN101735197 2010. (CA 153:116266)

${ }^{21}$ Kim, D. Y.; Kim, J. G.; Cho, D. J.; Lee, G. Y.; Kim, H. Y.; Woo, S. H.; Bae, W. C.; Lee, S. A.; Han, B. C. PCT Int. Appl. WO2004099186 2004. (CA 141:424204)

${ }^{22}$ Loiseleur, O.; Kaufmann, D.; Abel, S.; Buerger, H. M.; Meisenbach, M.; Schmitz, B.; Sedelmeier, G. PCT Int. Appl. WO2003066613 2003. (CA 139:180080)

${ }^{23}$ Kankan, R. N.; Rao, D. R. Brit. UK Pat. Appl. GB2398565 2004. (CA 141:207230)

${ }^{24}$ Gruza, H.; Mirek, S.; Jezewski, A.; Wrzosek, A. PCT Int. Appl. WO2010014022 2010. (CA 152:192169)

${ }^{25}$ Feng, L. Y.; Wang, C.; Bai, Y.; Han, N.; Jiao, J.; Qi, X. Org. Process Res. Develop. 2008, 12, 490. [CrossRef]

${ }^{26}$ Ivanov, A. S.; Shishkov, S. V. Monatsh Chem. 2009, 140, 619. [CrossRef]

${ }^{27}$ Liu, H.; Xia, W.; Luo, Y.; Lu, W. Monatsh Chem. 2010, 141, 907. [CrossRef]

${ }^{28}$ Leonetti, F.; Stefanachi, A.; Nicolotti, O.; Catto, M.; Pisani, L.; Cellamare, S.; Carotti, A. Curr. Med. Chem. 2011, 18, 2943. [CrossRef] [PubMed]

${ }^{29}$ Sítio da Secretária de Ciência e Tecnologia em Insumos Estratégicos (SCTIE). Disponível em:

$<$ http://portalsaude.saude.gov.br/portalsaud e/noticia/4544/162/investimentos-emlaboratorios-publicos-aumentam-5vezes.html>. Acesso em: 28 novembro 2012. ${ }^{30}$ Lombardino, J. G. U.S. US4,623,486 1986. (CA 106:50232)

${ }^{31}$ Rewcastle, G. W.; Denny, W. A.; Showalter, H. D. H. Curr. Org. Chem. 2000, 4, 679. [CrossRef]

${ }^{32}$ Feng, Z.; Chen, X.; Liu, H.; Meng, C.; Zuo, M. Faming Zhuanli Shenqing CN101423513 2009. (CA 151:8519) 